\title{
Comparative thermostability analysis of zoonotic and human influenza virus $A$ and $B$ neuraminidase
}

\author{
Vasily A. Evseenko ${ }^{1}$ - Svetlana V. Svyatchenko ${ }^{1} \cdot$ Natalia P. Kolosova $^{1} \cdot$ Valentina L. Kovrizhkina $^{1}$. \\ Vasiliy Y. Marchenko ${ }^{1}$ - Aleksander G. Durymanov ${ }^{1} \cdot$ Natalia I. Goncharova $^{1}$ · Alexander B. Ryzhikov ${ }^{1}$
}

Received: 3 July 2019 / Accepted: 12 October 2019 / Published online: 19 November 2019

(c) Springer-Verlag GmbH Austria, part of Springer Nature 2019

\begin{abstract}
Neuraminidase (NA) thermostability of influenza A and B viruses isolated from birds, swine and humans was measured to evaluate its variability associated with host body temperature. The highest $50 \%$ inactivation temperature $\left(\mathrm{IT}_{50}\right)$ was observed with $\mathrm{H} 3 \mathrm{~N} 8$ avian influenza virus $\left(74{ }^{\circ} \mathrm{C}\right)$, and the lowest $\mathrm{IT}_{50}$ was observed with the seasonal human $\mathrm{H} 3 \mathrm{~N} 2$ virus $\left(45.5^{\circ} \mathrm{C}\right)$. The $\mathrm{IT}_{50}$ values of $\mathrm{A}(\mathrm{H} 1 \mathrm{~N} 1) \mathrm{pdm} 09$ viruses $56.4-58.5^{\circ} \mathrm{C}$ were statistically higher than that of the prepandemic strain $\mathrm{A} /$ Solomon Islands/03/06 $\left(52.5^{\circ} \mathrm{C}\right)$. An analysis of $\mathrm{Ca}^{2+}$ binding sites revealed the correspondence of amino acid changes to NA thermostability. This study demonstrates that changes in NA thermostability correspond to differences in host body temperature.
\end{abstract}

\section{Introduction}

Wild waterfowl are natural reservoirs of influenza viruses [1]. Sixteen hemagglutinin (HA) and nine neuraminidase (NA) subtypes of influenza A virus (AIV), and most combinations of these, have been reported in wild birds. An exception to this may be recently reported the bat influenza viruses (H17N10 and H18N11), but to date these have not been associated with birds or with spillover to other hosts [2]. Periodically, genetic material from avian influenza viruses is mixed with genetic material from viruses infectious to humans in the process of reassortment. Human influenza virus strains with recently acquired avian surface and internal protein-encoding RNA segments were responsible for the pandemic influenza $\mathrm{A}(\mathrm{H} 2 \mathrm{~N} 2)$ outbreaks in 1957 and A(H3N2) outbreaks in 1968 [3, 4]. Swine are susceptible to infection with both avian and human virus strains, and various reassortants have been isolated from swine. Thus, swine have been proposed to be an intermediary in the process of emergence of reassorted viruses [5]. Besides virus receptor

Handling Editor: Carolina Scagnolari.

Vasily A. Evseenko

evseenko_va@vector.nsc.ru

1 State Research Center of Virology and Biotechnology

"Vector", Rospotrebnadzor, Koltsovo,

Novosibirsk Region 630559, Russian Federation differences between mammals and birds, there is another significant factor influencing virus reproduction: host body temperature. During flight, the body temperature of some birds can rise to $42-44^{\circ} \mathrm{C}$, while the body temperature of swine is $38-40^{\circ} \mathrm{C}$, and the normal temperature in humans is $36.6^{\circ} \mathrm{C}[6,7]$. Previous studies have shown that calcium is essential for the functioning and thermostability of influenza virus neuraminidase $[8,9]$.

Influenza virus neuraminidase is present as a tetramer in virus particles. Residues within the active site are highly conserved among all NA subtypes, including eight charged and polar residues (R118, D151, R152, R224, E276, R292, $\mathrm{R} 371$, and Y406) that have direct interaction with the substrate at the catalytic site. The geometry of the catalytic site is structurally stabilized through a network of hydrogen bonds and salt bridges formed by a constellation of largely conserved framework residues (E119, R156, W178, S179, D/N198, I222, E227, H274, E277, N294, and E425). Influenza NA possesses two confirmed calcium-ion binding sites and one putative calcium-ion binding site that was discovered in the pandemic $1918 \mathrm{~A}(\mathrm{H} 1 \mathrm{~N} 1)$ and $\mathrm{A}(\mathrm{H} 1 \mathrm{~N} 1) \mathrm{pdm} 09$ strains [10]. $\mathrm{Ca}^{2+}$ binding site I is formed by the four backbone carbonyl oxygens of D293, G297, G345, and N347, one of the carboxyl oxygens of D324, and a water molecule. The second calcium ion $\left(\mathrm{Ca}^{2+}\right.$ binding site II) is located at the fourfold axis of the NA tetramer and is coordinated by five water molecules. The four in-plane water molecules are stabilized by the symmetry-related D113 in an unidentate 
fashion, as well as by the main-chain carbonyl oxygen from K111 of a neighboring monomer. A putative $\mathrm{Ca}^{2+}$ binding site III, with pentagonal bipyramidal coordination, has been observed. The seven oxygen ligands involve the main-chain carbonyl oxygen of S389, the side chain carbonyl oxygen of N381, a carboxyl oxygen of monodentate D387, one bidentate D379, and two water molecules. This putative site is dependent on S319, P380, G382, W383 and T384 [10, 11].

The goal of this research study was to evaluate the extent of changes in neuraminidase thermostability in the context of influenza virus reassortment and evolutionary adaptation to various hosts. We measured the enzymatic thermostability of the neuraminidases of influenza viruses isolated from birds, swine and humans and performed mutational analysis of the neuraminidase genes.

Briefly, avian influenza A virus was isolated from infected embryonated eggs. Human influenza A and B viruses were isolated in the MDCK cell line [12]. Deep sequencing was performed using Illumina technology [13]. A list of viruses used in the study is presented in Table 1. In order to standardize the quantity of different viruses used in thermostability assessment, neuraminidase activity of viral stocks was determined prior to the experiment. For that, $30 \mu \mathrm{l}$ of $0.23 \mathrm{mM}$ fluorogenic substrate 2'-(4-methylumbelliferyl)$\alpha$-D-N-acetylneuraminic acid (MUNANA, Biosynth AG, Switzerland), which, when hydrolyzed by neuraminidase, releases the fluorescent product 4-methylumbelliferon, was added to an equal volume of a twofold dilution of the virus in $35 \mathrm{mM}$ 2-(N-morpholino)ethanesulfonic acid (MES) buffer ( $4 \mathrm{mM} \mathrm{CaCl}_{2}, 0.1 \% \mathrm{NP} 40$, pH 6.5). After 45 minutes of incubation at $37^{\circ} \mathrm{C}$, fluorescence intensity was measured at $448 \mathrm{~nm}$ (Infinite 200, Tecan). A virus dilution that corresponded to the middle of the linear part of the sigmoid curve was chosen for the thermostability assessment experiment. Aliquots of diluted viruses were incubated in a gradient thermal cycler (Bio-Rad) for 30 minutes in the temperature range of $40-80{ }^{\circ} \mathrm{C}$, followed by the incubation at $37{ }^{\circ} \mathrm{C}$ with MUNANA substrate for 45 minutes, after which fluorescence intensity was measured. Residual neuraminidase activity of heated aliquots was expressed as a percentage of the fluorescence intensity in a reaction with an unheated aliquot stored at $4{ }^{\circ} \mathrm{C}$ prior to neuraminidase activity determination. The temperature at which incubation for 30 minutes led to a $50 \%$ decrease in the residual neuraminidase activity was designated as the " $50 \%$ inactivation temperature" $\left(\mathrm{IT}_{50}\right)$. This parameter was used to compare the thermostability of different viruses. The $\mathrm{IT}_{50}$ value was determined as the mean of three independent experiments with three replicates each [14]. The mean, standard deviation, and confidence interval were calculated in Excel (Microsoft). First, a subtype-specific alignment of neuraminidase amino acid sequences translated in silico was performed using MEGA 6.0 software, and focal positions and motifs of the catalytic domain and $\mathrm{Ca}^{2+}$ binding sites were identified for N2 neuraminidases. Then multiple alignments of all sequences were performed using the protein BLAST algorithm, using NCBI data resources. The amino acid numbering used in Table 1 corresponds to the $\mathrm{N} 2$ sequence. Catalytic site and framework residues were identical in all studied strains, taking into account the initial length of the protein and specific deletions (with the exception of A/Texas/12/2007 (H3N2), which has an E119V mutation in the framework region that is associated with resistance to neuraminidase inhibitors, and A/Bethesda/956/2006 (H3N2), with an R292K substitution in the catalytic site that is also associated with resistance to neuraminidase inhibitors). The numbering of these amino acids and the distances between the amino acids were used as a basis for the alignment, and potential $\mathrm{Ca}^{2+}$ binding sites were located in the NA sequences (Table 1). It is notable that the difference between the highest and lowest NA IT 50 values among the studied influenza viruses was $28.5^{\circ} \mathrm{C}$. The highest NA $\mathrm{IT}_{50}$ was observed for an $\mathrm{H} 3 \mathrm{~N} 8$ avian influenza virus $\left(74^{\circ} \mathrm{C}\right)$, and the lowest was observed for a seasonal human $\mathrm{H} 3 \mathrm{~N} 2$ virus $\left(45.5^{\circ} \mathrm{C}\right)$. Overall, NA $\mathrm{IT}_{50}$ values for the avian viruses varied from 57.7 to $74{ }^{\circ} \mathrm{C}$, NA IT $_{50}$ values for the human type $\mathrm{B}, \mathrm{H} 3 \mathrm{~N} 2$ and prepandemic $\mathrm{A} / \mathrm{H} 1 \mathrm{~N} 1$ viruses varied from 45.5 to $54.7{ }^{\circ} \mathrm{C}$, and NA IT ${ }_{50}$ values for $\mathrm{A}(\mathrm{H} 1 \mathrm{~N} 1) \mathrm{pdm} 09$ were in an intermediate position, varying from 56.4 to $58.5{ }^{\circ} \mathrm{C}$. Among the $\mathrm{N} 1$ influenza viruses, it is important to note the higher IT $_{50}$ values measured for the pandemic $\mathrm{A}(\mathrm{H} 1 \mathrm{~N} 1) \operatorname{pdm} 09$ viruses $\left(56.4\right.$ to $\left.58.5^{\circ} \mathrm{C}\right)$ compared to prepandemic strain $\mathrm{A} / \mathrm{Solomon}$ Islands/03/06 $(\mathrm{H} 1 \mathrm{~N} 1)\left(52.5^{\circ} \mathrm{C}\right)$. A statistically significant $(P=0.95)$ average difference in $\mathrm{IT}_{50}$ of $5.07{ }^{\circ} \mathrm{C}$ was observed. At the same time, there was no statistically significant $(P=0.95)$ difference in $\mathrm{IT}_{50}$ values between the pandemic $\mathrm{A}(\mathrm{H} 1 \mathrm{~N} 1)$ pdm09 strains and the highly pathogenic avian influenza (HPAI) $\mathrm{H} 5 \mathrm{~N} 1$ virus. Analysis of the three $\mathrm{Ca}^{2+}$ binding sites revealed that all of them had substitutions among the studied influenza viruses (Table 1).

Among the studied N1 viruses, the substitutions $\mathrm{D} 347 \mathrm{~N} / \mathrm{Y}$ in $\mathrm{Ca}^{2+}$ binding site I and the substitutions N385G and D389N/K/E in putative $\mathrm{Ca}^{2+}$ binding site III were investigated previously using reverse genetics in the study focusing on NA stability [15]. N385G and D389N substitutions were found in A/California/04/2009 (H1N1)pdm09 when compared to A/Solomon Islands/03/06 (H1N1), and together with the observed D347N substitution, these may be associated with increased NA thermostability. More recently isolated A(H1N1)pdm09 viruses (2018-2019) had amino acid substitutions in the investigated positions similar to those in A/California/04/2009 (H1N1)pdm09 when compared to the studied prepandemic A/Solomon Islands/03/06 (H1N1) strain, and their thermostability was similar to that of $\mathrm{A} / \mathrm{Cal}-$ ifornia/04/2009 (H1N1)pdm09 NA. The avian strain A/rook/ Chany/32/2015 (H5N1) has the substitutions D347Y and 


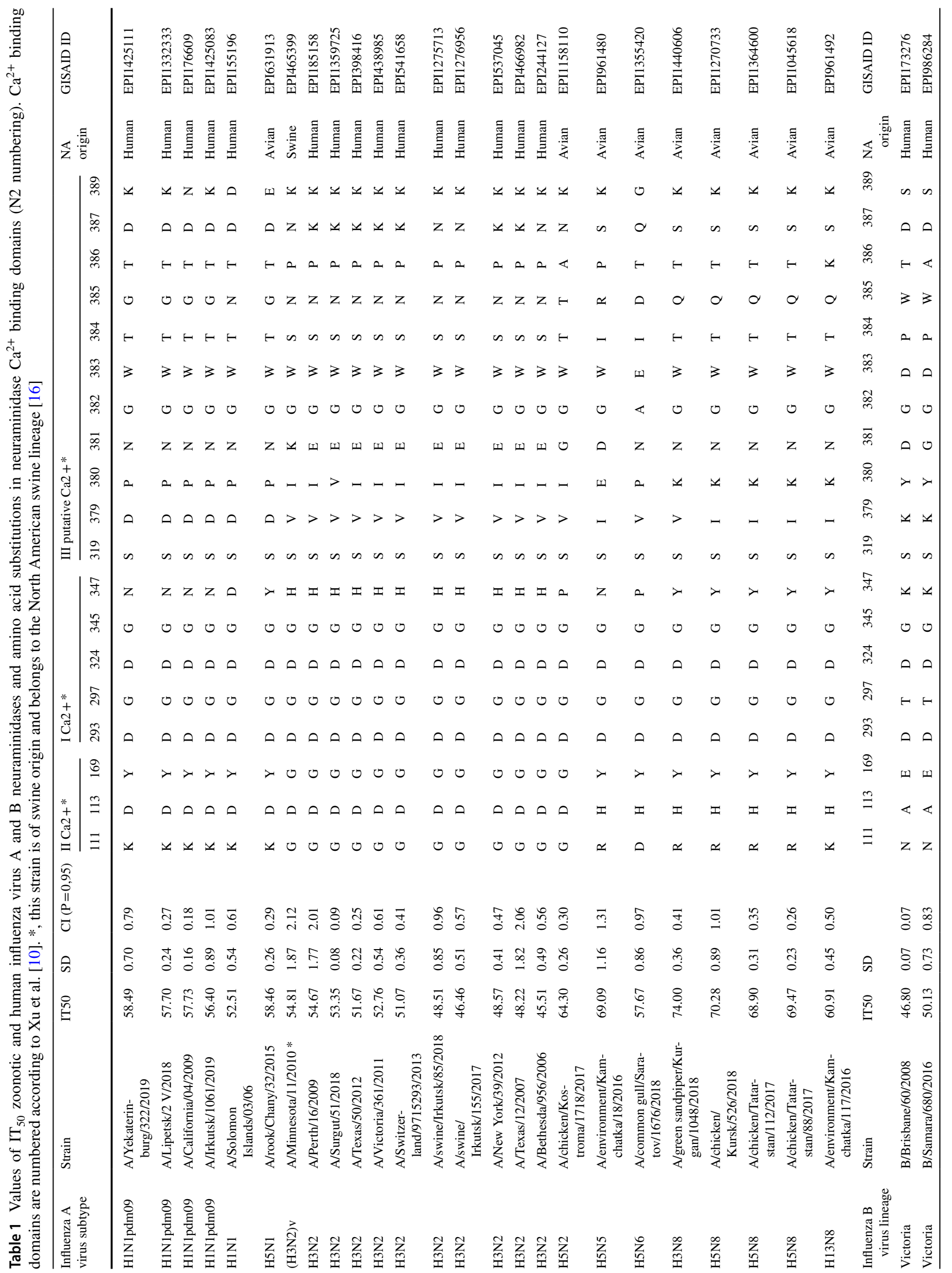




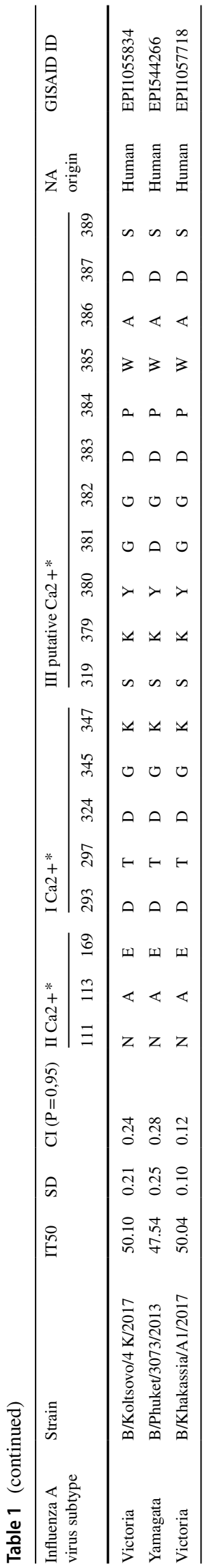

D389E. It is worth noting that A/Solomon Islands/03/06-like viruses have been in circulation for several decades in the human population and have undergone adaptation human hosts, which was possibly associated with a decrease in the thermostability of the NA of these viruses.

Among the N2 influenza viruses studied, the maximum average difference in the $\mathrm{IT}_{50}$ value was $18.79{ }^{\circ} \mathrm{C}$ (A/Bethesda/956/2006 (H3N2) and A/chicken/Kostroma/1718/2017 (H5N2)). Among the seasonal human influenza viruses the average $\mathrm{IT}_{50}$ difference was $9.3^{\circ} \mathrm{C}(\mathrm{A} /$ Bethesda/956/2006 (H3N2) and A/Perth/16/2009 (H3N2)). It was shown earlier by reverse genetics that the substitutions R292K, found in A/Bethesda/956/2006 (H3N2), and E117V, found in $\mathrm{A} /$ Texas/12/2007 (H3N2), decrease neuraminidase thermostability [17]. A/chicken/Kostroma/1718/2017 (H5N2) is an HPAI virus, and its neuraminidase has a deletion of 23 amino acids in the stalk region. Only A/chicken/ Kostroma/1718/2017 (H5N2) has an H347P substitution in $\mathrm{Ca}^{2+}$ binding site $\mathrm{I}$. $\mathrm{Ca}^{2+}$ binding site II was identical in the $\mathrm{N} 2$ viruses studied. The putative $\mathrm{Ca}^{2+}$ binding site III is more variable, and the characteristic amino acid substitutions E381G, S384T, N385T, and P386A were found in A/chicken/Kostroma/1718/2017 (H5N2). Several of these viruses had IT $_{50}$ values identical to those of human Influenza B viruses. Genetic BLAST analysis (GISAID) of the swine A/swine/Irkutsk/85/2018 (H3N2) and A/swine/ Irkutsk/155/2017 (H3N2) viruses showed that they are reassortants of the human seasonal $\mathrm{A}(\mathrm{H} 3 \mathrm{~N} 2)$ and $\mathrm{A}(\mathrm{H} 1 \mathrm{~N} 1)$ pdm09 influenza viruses and their $\mathrm{N} 2$ genes are $95 \%$ identical to that of A/Netherlands/2013/2003 (H3N2).

All of the N8 viruses studied were isolated from birds. The maximum difference in $\mathrm{IT}_{50}$ values was $13.9^{\circ} \mathrm{C}$ and it was detected when comparing A/green sandpiper/ Kurgan/1048/2018 (H3N8) and A/environment/Kamchatka/117/2016 (H13N8). The NA of A/green sandpiper/ Kurgan/1048/2018 (H3N8) has the distinctive substitution I379V, and A/environment/Kamchatka/117/2016 (H13N8) has T386K. All of the influenza A/H5N8 viruses tested had identical amino acids in all three $\mathrm{Ca}^{2+}$ binding sites and nearly identical $\mathrm{IT}_{50}$ values.

The influenza B viruses of the Yamagata and Victoria lineages that were used in this study are highly adapted human influenza viruses. The influenza $\mathrm{B}$ viruses had $\mathrm{IT}_{50}$ values in the range of $46.8-50.13{ }^{\circ} \mathrm{C}$ and were statistically indistinguishable from prepandemic A/Solomon Islands/03/06 (H1N1). Analysis of $\mathrm{Ca}^{2+}$ binding sites revealed the amino acid substitution D381G in the putative $\mathrm{Ca}^{2+}$ binding site III, which can be associated with an average IT $_{50}$ increase of $2.92{ }^{\circ} \mathrm{C}$, and it is likely to be involved in the increase in NA thermostability.

Genome sequencing performed in our study revealed amino acid substitutions in the NA of influenza viruses from a variety of hosts that are potentially associated with 
influenza neuraminidase thermostability. Further investigation of potential $\mathrm{Ca}^{2+}$ binding sites, which vary among the studied NA subtypes, is necessary for identification of substitutions that are significant for the NA thermostability of specific neuraminidases. Influenza viruses have high genetic variability and are subjected to evolutionary selection. Analysis of the dynamics of viral quasispecies populations has revealed that virus variants with lower fitness can outcompete viruses with higher fitness. Thus, the selection may favor a virus that is not excessively harmful to its natural host [18]. For influenza virus with an avian-type neuraminidase that is in the process of adaptation to persistence in the human population, excessively high thermostability may be a negative factor for selection. In cases of influenza disease, the human body temperature may rise to $40-41^{\circ} \mathrm{C}$, which potentially may have less effect on a more stable viral neuraminidase and presumably could lead to a more severe outcome, which would be unfavorable for long-term virus persistence in the host. Consistent with quasispecies theory [18], a high mutation rate could have produced a virus with a less thermostable neuraminidase that outcompetes a virus variant with a more thermostable neuraminidase. In nature, increased thermostability is beneficial for persistence of influenza viruses in the environment, but it may not be selected and sustained during the process of adaptation to human hosts once the virus crosses the species barrier.

Highly pathogenic avian influenza H5N1 virus has a polybasic cleavage site in its $\mathrm{H} 5$ hemagglutinin. This feature allows the virus to reproduce in various organs [19]. At present, this polybasic site is a key marker of high pathogenicity for birds and mammals. We believe that our data on neuraminidase thermostability may complement the understanding of pathogenesis of HPAI in mammals. The pathogenicity of a newly emerging pandemic influenza virus can be explained not only by the absence of population immunity but also by specific features of the virus, such as its polymerase complex and, possibly, its genetically determined neuraminidase thermostability. We suggest that the NA $\mathrm{IT}_{50}$ may be considered an adaptation marker that indicates that the pandemic influenza virus is in an adaptation phase. This hypothesis needs to be further investigated by performing neuraminidase thermostability analysis on viruses at the beginning of adaptation to a new host (e.g., the original A/ Hong Kong/68 (H3N2) and subsequent A/H3N2 seasonal human viruses).

Evaluation of influenza virus NA thermostability across different hosts revealed a remarkable range of NA thermostability in avian, swine, and human influenza viruses. Our hypothesis suggests that the high NA thermostability observed in avian influenza viruses may be necessary for persistence in the environment and optimal functioning in birds, which have a higher body temperature than humans. Our study has possibly revealed significant links between virus adaptation to human hosts and a decrease in NA thermostability, which was particularly evident in the case of the comparison of the prepandemic $A(\mathrm{H} 1 \mathrm{~N} 1)$ of 2006, with a long adaptation history, to the more recently emerged $\mathrm{A}(\mathrm{H} 1 \mathrm{~N} 1)$ pdm09 viruses. Alternative hypotheses may explain the observed changes in neuraminidase thermostability by variable environmental pressure or other factors. Genetic analysis showed a correlation of amino acid substitutions in NA $\mathrm{Ca}^{2+}$ binding sites with changes in NA thermostability in some cases and revealed new potentially important amino acid substitutions. Our study demonstrated that thermostability of neuraminidase, which is easily assessed by measuring the NA $\mathrm{IT}_{50}$, may be considered as an additional marker of the adaptation phase of pandemic influenza virus and needs to be considered for its possible contribution to the pandemic potential of influenza viruses.

Acknowledgements The authors thank the CDC (GA, USA), WHO CC for Reference and Research on Influenza, Australia, and Crick Worldwide Influenza Centre, UK, for providing virus strains through IRR and for sharing sequences through GISAID. The authors are especially grateful to the SRC VB "Vector" core sequence facilities.

Funding This research was funded by the Russian Federal Service for Surveillance on Consumer Rights Protection and Human Wellbeing (Rospotrebnadzor).

\section{Compliance with ethical standards}

Conflict of interest Evseenko V.A. declares that he has no conflict of interest. Kolosova N.P. declares that she has no conflict of interest. Svyatchenko S.V. declares that she has no conflict of interest. Kovrizhkina V.L. declares that she has no conflict of interest. Durymanov A.G. declares that he has no conflict of interest. Goncharova N.A. declares that she has no conflict of interest. Marchenko V.Y. declares that he has no conflict of interest. Ryzhikov A.B. declares that he has no conflict of interest.

Ethical approval All applicable international, national and institutional guidelines for the care and use of animals were followed. This article does not contain any studies with human participants performed by any of the authors.

\section{References}

1. Webster RG, Bean WJ, Gorman OT, Chambers TM, Kawaoka Y (1992) Evolution and ecology of influenza A viruses. Microbiol Rev 56(1):152-179

2. Tong S, Zhu X, Li Y, Shi M, Zhang J, Bourgeois M et al (2013) New World bats harbor diverse influenza A viruses. PLoS Pathog 9:e1003657. https://doi.org/10.1371/journal.ppat.1003657

3. Kawaoka Y, Krauss S, Webster RG (1989) Avian-to-human transmission of the PB1 gene of influenza A viruses in the 1957 and 1968 pandemics. J Virol 63(11):4603-4608

4. Scholtissek C, Rohde W, Von Hoyningen V, Rott R (1978) On the origin of the human influenza virus subtypes H2N2 and H3N2. Virology 87(1):13-20 
5. Scholtissek C (1994) Source for influenza pandemics. Eur J Epidemiol 10(4):455-458

6. Torre-Bueno JR (1976) Temperature regulation and heat dissipation during flight in birds. J Exp Biol 65:471-482

7. Murray Pullar E (1949) The rectal temperature in normal and infected pigs. Br Vet J 105(12):437-453

8. Burmeister W, Cusack S, Ruigrok RJ (1994) Calcium is needed for the thermostability of influenza B virus neuraminidase. Gen Virol 75(2):381-388. https://doi.org/10.1099/0022-1317-75-2-381

9. Baker NJ, Gandhi SS (1976) Effect of $\mathrm{Ca}++$ on the stability of influenza virus neuraminidase. Arch Virol 52(1-2):7-18

10. Xu X, Zhu X, Dwek RA, Stevens J, Wilson IA (2008) Structural characterization of the 1918 influenza virus H1N1 neuraminidase. J Virol 82(21):10493-10501. https://doi.org/10.1128/JVI.00959 $-08$

11. Air GM (2012) Influenza neuraminidase. Influenza Other Respir Viruses 6(4):245-256

12. Manual for the laboratory diagnosis and virological surveillance of influenza. 2011 (ISBN: 9789241548090)

13. Marchenko V, Goncharova N, Susloparov I, Kolosova N, Gudymo A, Svyatchenko S et al (2018) Isolation and characterization of H5Nx highly pathogenic avian influenza viruses of clade 2.3.4.4 in Russia. Virology 525:216-223

14. Tewawong N, Marathe BM, Poovorawan Y, Vongpunsawad S, Webby RJ, Govorkova EA (2018) Neuraminidase inhibitor susceptibility and neuraminidase enzyme kinetics of human influenza
A and B viruses circulating in Thailand in 2010-2015. PLoS One 13(1):e0190877

15. Labadie T, Batéjat C, Manuguerra JC, Leclercq I (2018) Influenza virus segment composition influences viral stability in the environment. Front Microbiol 9:1496. https://doi.org/10.3389/fmicb .2018.01496 (Published 2018 Jul 9)

16. Jhung MA, Epperson S, Biggerstaff M et al (2013) Outbreak of variant influenza $\mathrm{A}(\mathrm{H} 3 \mathrm{~N} 2)$ virus in the United States. Clin Infect Dis 57(12):1703-1712. https://doi.org/10.1093/cid/cit649

17. Yen HL, Herlocher LM, Hoffmann E et al (2005) Neuraminidase inhibitor-resistant influenza viruses may differ substantially in fitness and transmissibility. Antimicrob Agents Chemother 49(10):4075-4084. https://doi.org/10.1128/ AAC.49.10.4075-4084.2005

18. Negovetich NJ, Webster RG (2010) Thermostability of subpopulations of $\mathrm{H} 2 \mathrm{~N} 3$ influenza virus isolates from mallard ducks. J Virol 84(18):9369-9376. https://doi.org/10.1128/JVI.01170-10

19. Belser JA, Tumpey TM (2013) H5N1 pathogenesis studies in mammalian models. Virus Res 178(1):168-185. https://doi. org/10.1016/j.virusres.2013.02.003

Publisher's Note Springer Nature remains neutral with regard to jurisdictional claims in published maps and institutional affiliations. 